\title{
Differences Between Doped and Undoped Zirconium Alloy Oxide Layers
}

\author{
H. Frank
}

\begin{abstract}
Two samples of undoped Zr1Nb and of Zry- $4 \mathrm{~W}$, doped with additives of Sn, Fe, Cr, were oxidized for 42 days at $360{ }^{\circ} \mathrm{C}$, forming oxide layers $1.9 \mu \mathrm{m}$ in thickness. The I-V characteristics, measured at room temperature up to $160^{\circ} \mathrm{C}$, were used to compare the transport properties in order to assess the influence of doping. In both samples, the activation energy was equal to $1.2 \mathrm{eV}$ and the temperature dependence of resistivity, electron mobility and carrier concentration, and also the behavior of injection and extraction currents, were found to be equal inside the error limits, thus proving the doping to be ineffective. Zirconium oxide fits into the group of oxide semiconductors, being an n-type reduction semiconductor, conduction depending on stoichiometric deviation, i.e. missing oxygen.
\end{abstract}

Keywords: Zirconium alloy oxide layers, doped and undoped samples, I-V characteristics, temperature dependence of transport parameters, activation energy, injection and extraction currents and their time dependence, reduction semiconductor.

\section{Introduction}

In normal atomic semiconductors, e.g. Si, the conductivity is sensitive to doping with atoms of different valency, whereas in oxidic semiconductors conductivity depends rather on stoichiometric deviations and less on doping [1]. It is the aim of this work to assess the influence of alloying atoms acting as dopants in oxide layers. There were two samples of undoped Zr1Nb (alloying with $1 \%$ of Niobium does not introduce doping centers), and of Zry-4W without $\mathrm{Nb}$ but alloyed with $\mathrm{Sn}, \mathrm{Fe}, \mathrm{Cr}$, of $1.46,0.2,0.1$ wt \%, respectively.

Two tube specimens $30 \mathrm{~mm}$ in length and $9 \mathrm{~mm}$ in outer diameter were oxidized under VVER conditions (Tab. 1).

\section{Experimental}

The oxide on the end faces of the tubes was ground off for good contact. Painted-on electrodes of colloidal silver $6.0 \mathrm{~mm}$ in diameter were used. The samples were mounted into a mini-thermostat for measurement at temperatures up to $160{ }^{\circ} \mathrm{C}$. Details of the measuring procedure are given in [2]. The relative permittivity was calculated from the measured electrode capacity at $1000 \mathrm{~Hz}$ with known geometrical factors. The levels were extremely low, 12.4 and 8.3, for D 61 and U 71, respectively. The V-I characteristics were symmetrical, therefore only the forward voltage branch, with the positive terminal connected to the zirconium metal, was measured with voltage steps of $0.5 \mathrm{~V}$ up to $7 \mathrm{~V}$, and at constant temperatures in steps of $20^{\circ} \mathrm{C}$, or with continually increasing temperatures.

At lower temperatures, the samples had normal I-V characteristics of the form of eq. 1 with space-charge limited currents, which tended to become linear at higher temperatures.

$$
I=a U^{2}+b U+c,
$$

Eq. 1 can be used to compute resistivity $\rho$, mobility $\mu$ and carrier concentration $n$. Further details concerning theoretical aspects are given in $[3,4]$.

\section{$3 \quad$ Results and discussions}

In order to stabilize the colloidal Ag contacts, it was necessary to anneal the layers at a temperature of over $100{ }^{\circ} \mathrm{C}$. The temperature was continually increased, and at each rise of $5{ }^{\circ} \mathrm{C}$ the zero current was recorded. At $140{ }^{\circ} \mathrm{C}$ the temperature was again decreased. In both samples, the zero current was constant with about $12 \mathrm{pA}$ up to $80^{\circ} \mathrm{C}$ and then increased to over $1000 \mathrm{pA}$ at $140^{\circ} \mathrm{C}$. At second heating, the zero current grew steadily in exponential form. Current measured with constant voltage of $0.5 \mathrm{~V}$ and continually increasing temperature gave straight lines, allowing computation of the activation energies. In $\mathrm{D} 61,1.19 \mathrm{eV}$

Table 1: Characterization of Samples

\begin{tabular}{|cc|c|c|c|c|c|}
\hline Sample number & type & Short name & Medium & Temperature $\left({ }^{\circ} \mathrm{C}\right)$ & Time $(\mathrm{d})$ & Thickness $(\mu \mathrm{m})$ \\
\hline E 110G 6136268 & Zry-4W & D 61 (doped) & VVER & 360 & 42 & 1.91 \\
\hline E 110 7136108 & Zr1Nb & U 71 (undoped) & VVER & 360 & 42 & 1.90 \\
\hline
\end{tabular}




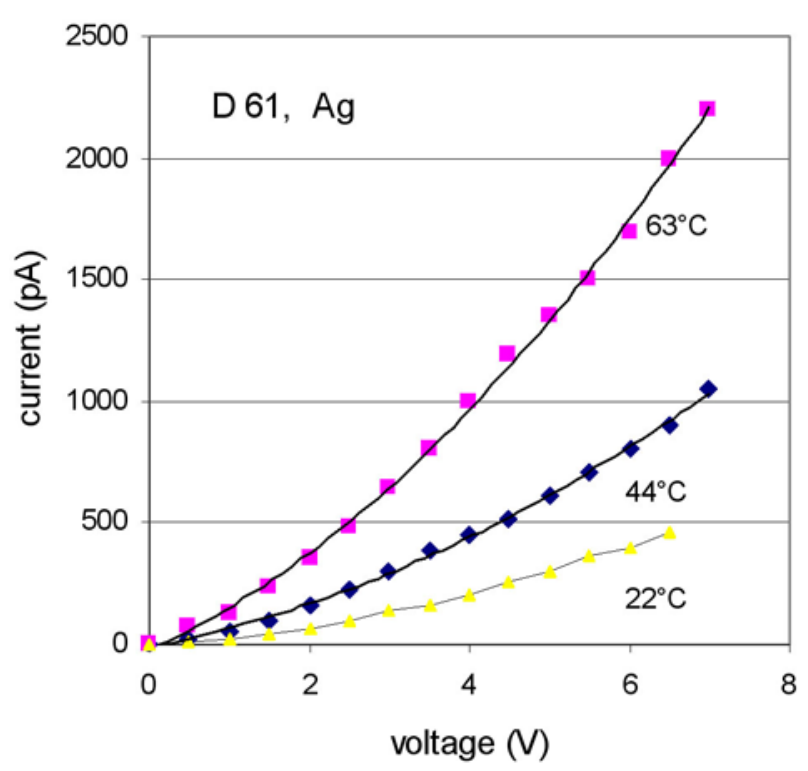

Fig. 1: Temperature dependence of I-V characteristics, D 61 doped

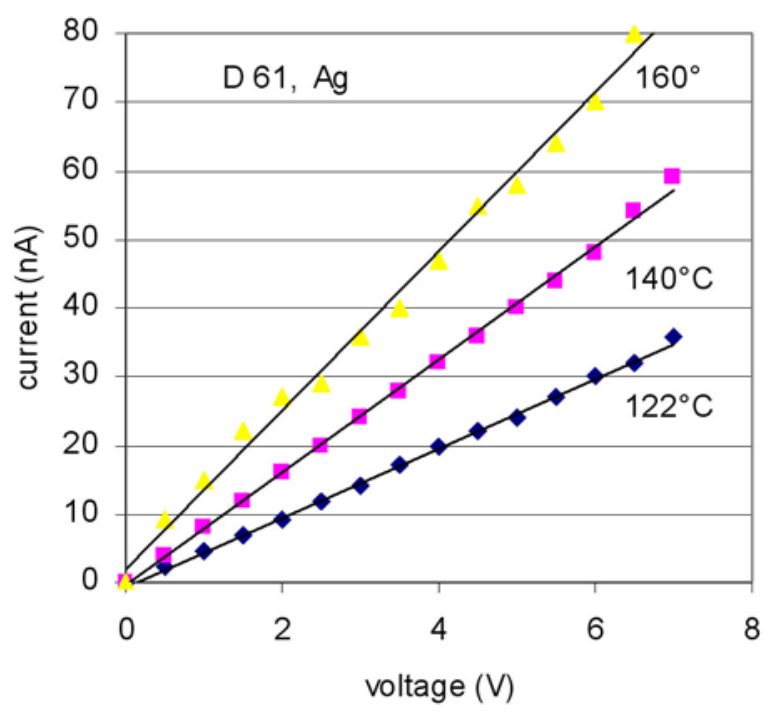

Fig. 3: Linear I-V characteristics at higher temperatures of D 61 doped

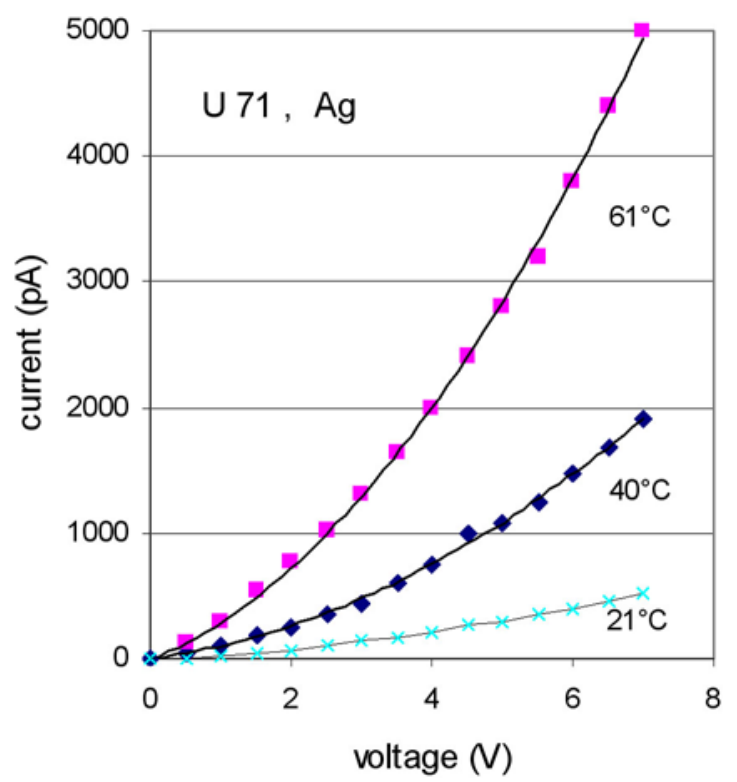

Fig. 2: Temperature dependence of I-V characteristics, U 71 undoped

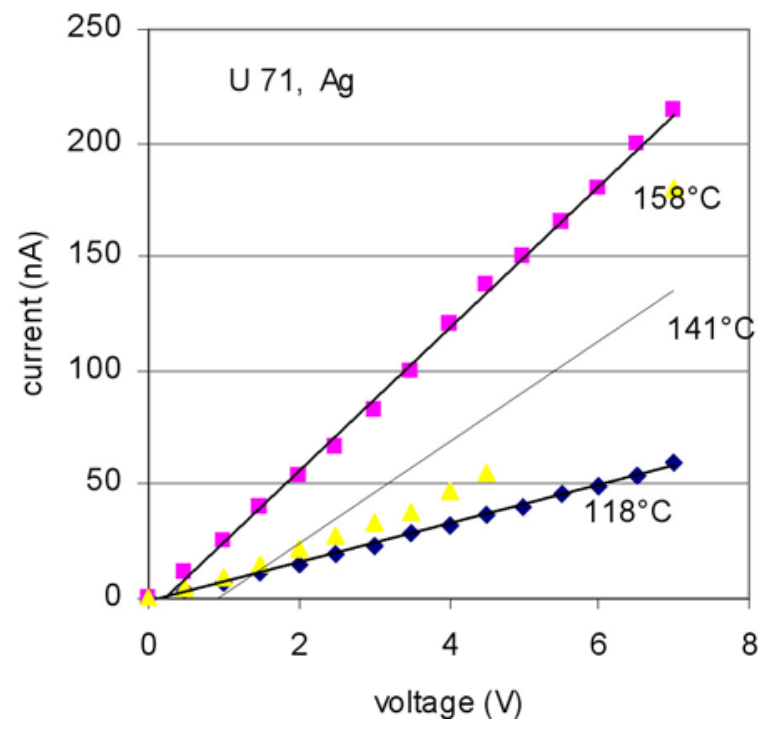

Fig. 4: As in Fig. 3, U 71 undoped

Table 2: Parameters of both samples at room temperature (Figs. 3, 4)

\begin{tabular}{|ccccc|}
\hline Sample & Thickness $(\mu \mathrm{m})$ & Resistivity $(\Omega \mathrm{cm})$ & Mobility $\left.\left(\mathrm{cm}^{2}\right) / \mathrm{Vs}\right)$ & Concentration $\left(\mathrm{cm}^{-3}\right)$ \\
\hline D 61 & 1.9 & $11.3 \cdot 10^{14}$ & $2.4 \cdot 10^{-10}$ & $2.1 \cdot 10^{14}$ \\
U 71 & 1.90 & $1.07 \cdot 10^{14}$ & $2.8 \cdot 10^{-10}$ & $2.1 \cdot 10^{14}$ \\
\hline
\end{tabular}

was found for increasing temperature, and $1.32 \mathrm{eV}$ for decreasing temperature. Similar behavior in U 71 gave $1.10 \mathrm{eV}$ and $1.31 \mathrm{eV}$, respectively. Taking timedependent deviations into account, the middle value of both samples is $1.2 \mathrm{eV}$.

Due to the small thickness, the critical field strength is achieved already at about $1 \mathrm{~V}$, and there- fore the I-V characteristics were measured only up to $7 \mathrm{~V}$, and were found to be very similar in both samples.

The characteristics (Figs. 1, 2) of both samples are very similar, and their values are shown in Tab. 2 .

The I-V characteristics measured at constant temperatures were taken as base values for assessing resistivity, mobility, carrier concentration and activation 


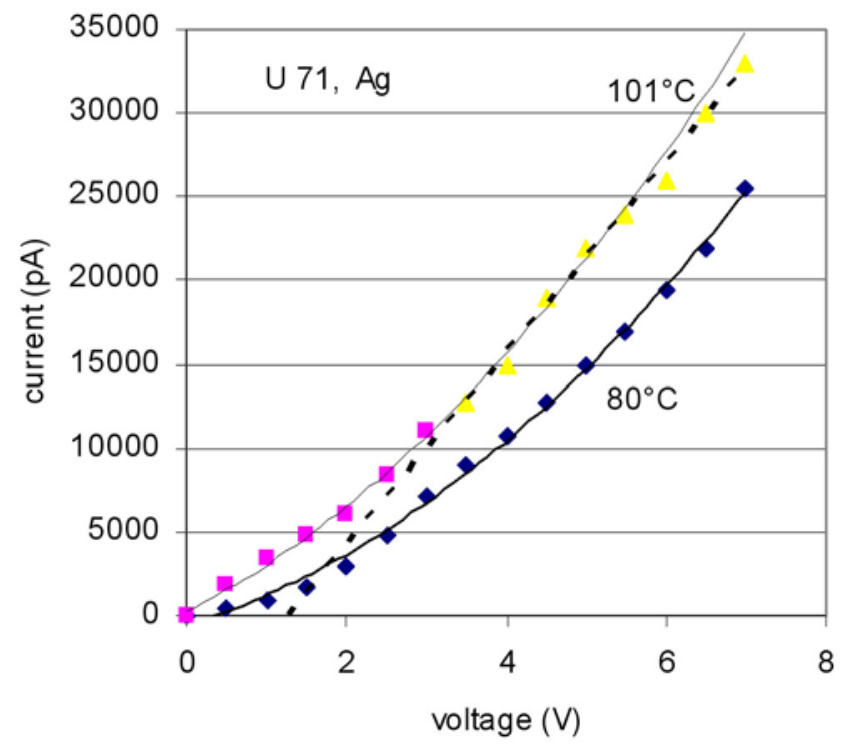

Fig. 5: At $100{ }^{\circ} \mathrm{C}$ (and higher), the current rises in a linear way (beginning at $3.5 \mathrm{~V}$ )

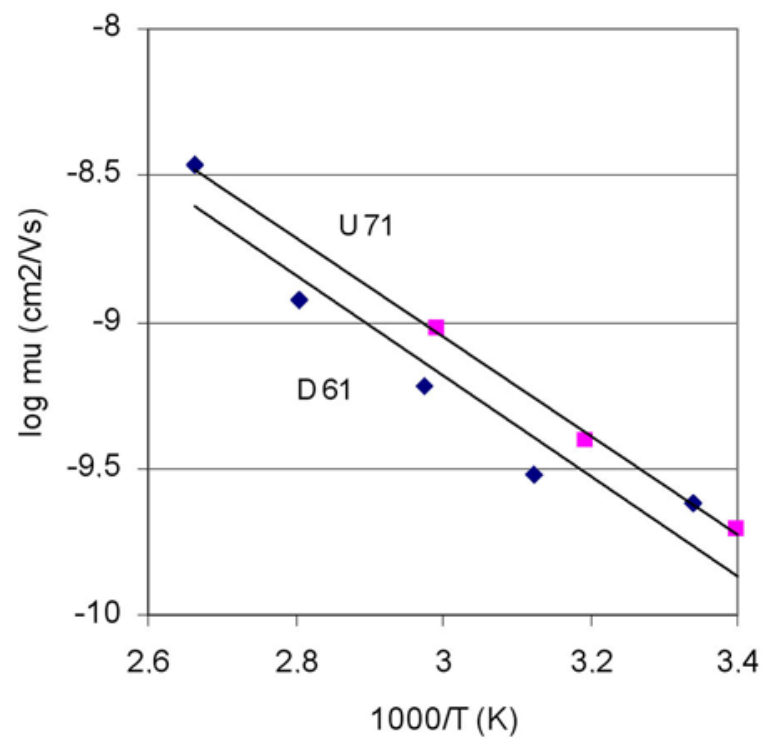

Fig. 7: Equal temperature dependence of mobility in both samples

energy. As can be seen, comparing Figs. 1-4, the behavior of both samples is very similar, at lower temperatures up to $80^{\circ} \mathrm{C}$, eq. 1 is obeyed, creating second order curves, whereas at higher temperatures linear dependence of current on voltage is observed. The change from quadratic to linear form is shown in Fig. 5, where at $101^{\circ} \mathrm{C}$ the change takes place at $3.5 \mathrm{~V}$.

Plotting $\log \rho$ as a function of the reciprocal absolute temperature in Fig. 6 gives the same activation energy of $1.2 \mathrm{eV}$, proving the ineffectiveness of doping.

The similarity of the two samples is confirmed by the practically identical temperature dependence.

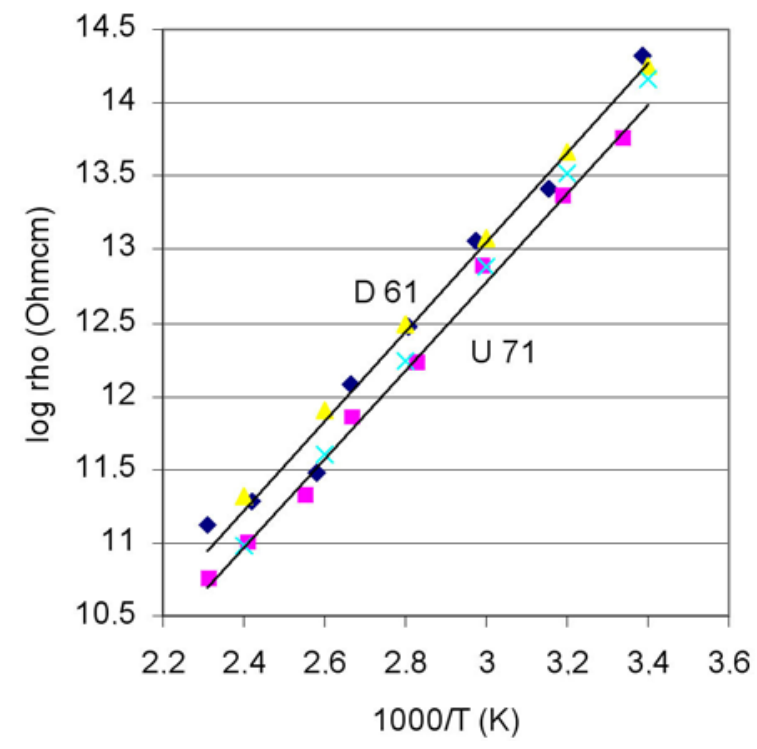

Fig. 6: Temperature dependence of resistivity using data of I-V characteristics, (each sample measured twice to prove reproducibility), activation energy of resistivity equal in both samples

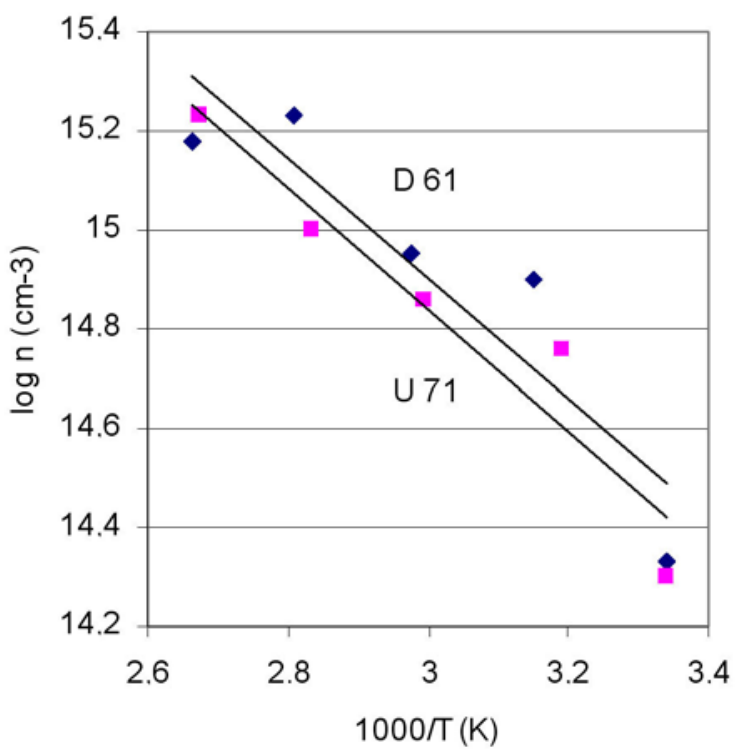

Fig. 8: Equal temperature dependence of carrier concentration in both samples

The time dependence of the injection and extraction currents was compared in the two samples, and was found at room and higher temperatures to be very nearly equal, as can be seen in Figs. 9, 10.

As shown in Figs. 9, 10, on application of voltage a high starting current, injecting most of the carriers, gradually diminishes, building up a space charge, until a stable equilibrium current is reached after a certain time, obeying eq. 1 . When the contacts with the picoamperemeter are shortened, the injected space charge flows out and gives rise to a negative extraction current, which is equal to the former (positive) injection 


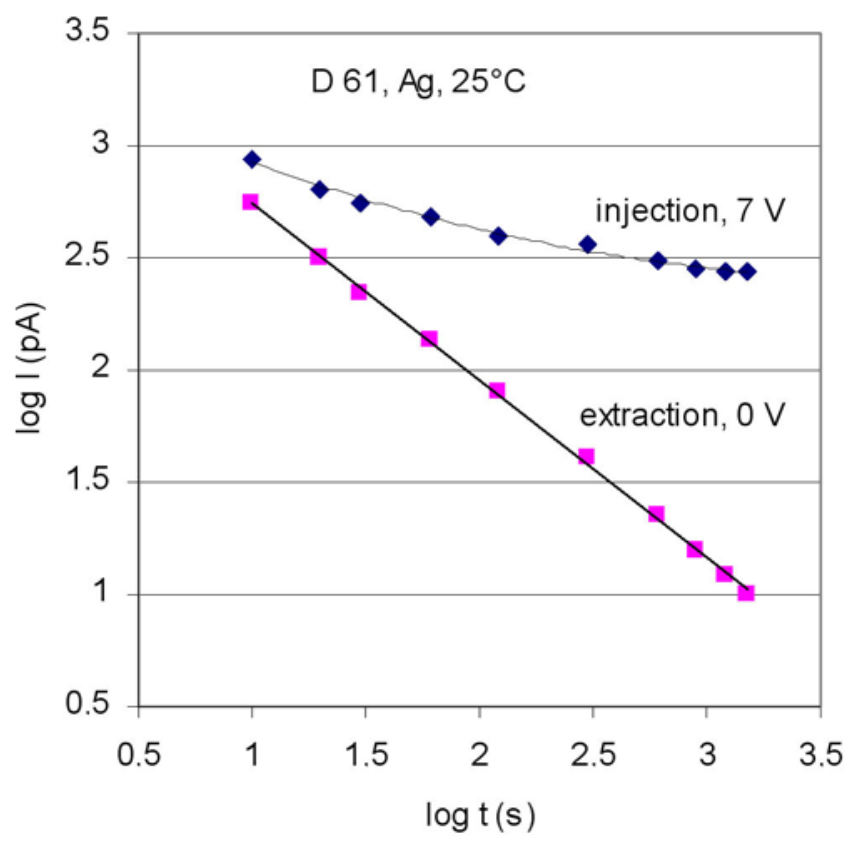

Fig. 9: D 61, time dependence of injection current after application of constant voltage, and of extraction current measured without driving voltage, electrodes shorted, using a pico-amperemeter

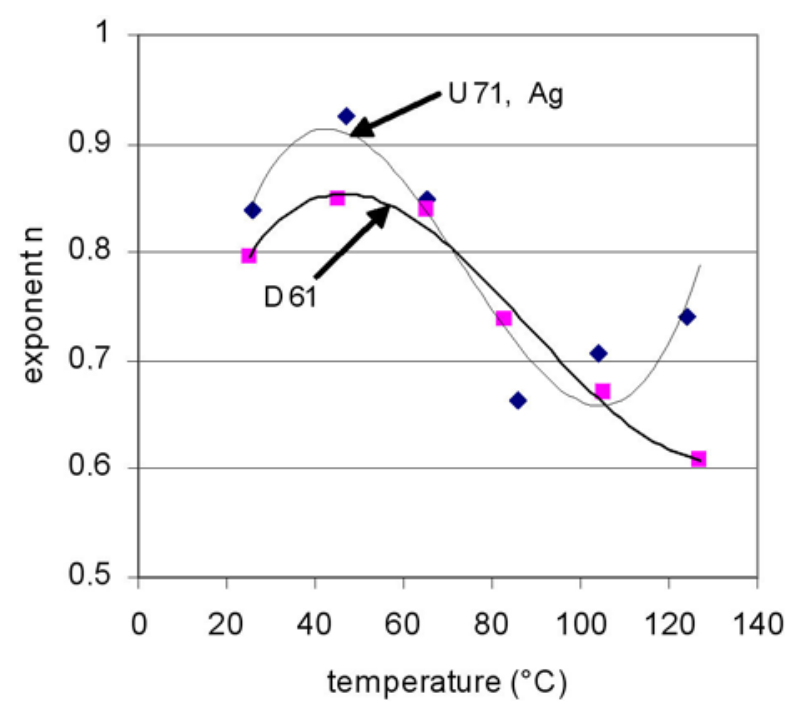

Fig. 11: Comparison of temperature dependent exponents $n$

current and obeys the power law of eq. $2[5]$

$$
I=B t^{-n},
$$

with time $t$ and exponent $n<1$. The extracted charge $Q$ can be computed by integration,

$$
Q=\int_{t_{1}}^{t_{2}} B t^{-n} \mathrm{~d} t=\frac{B\left(t_{2}^{1-n}-t_{1}^{1-n}\right)}{1-n}
$$

The slopes of the straight lines in Figs. 9, 10 give the

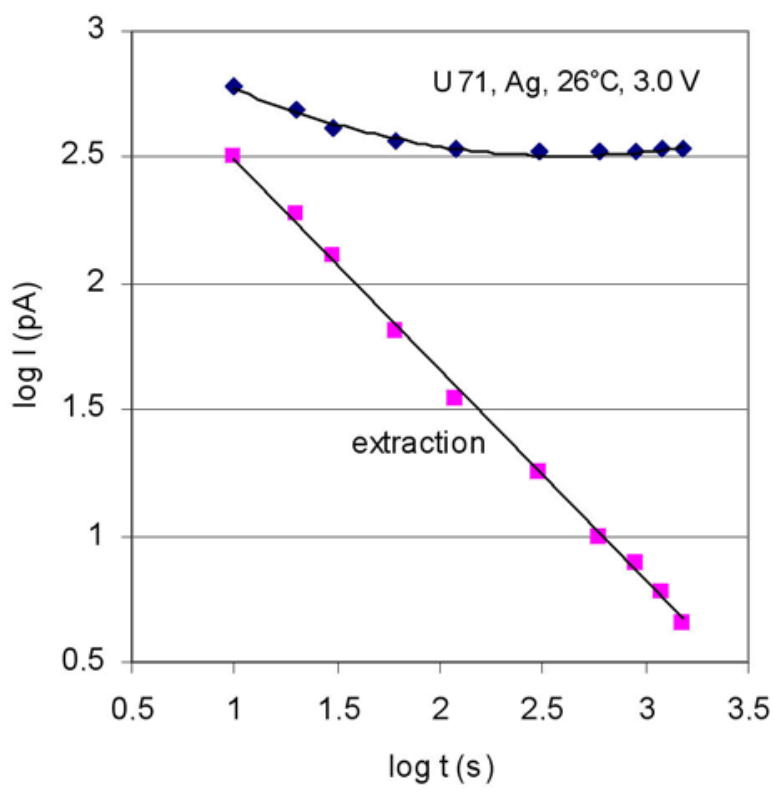

Fig. 10: U 71, as in Fig. 9, with lower injection voltage

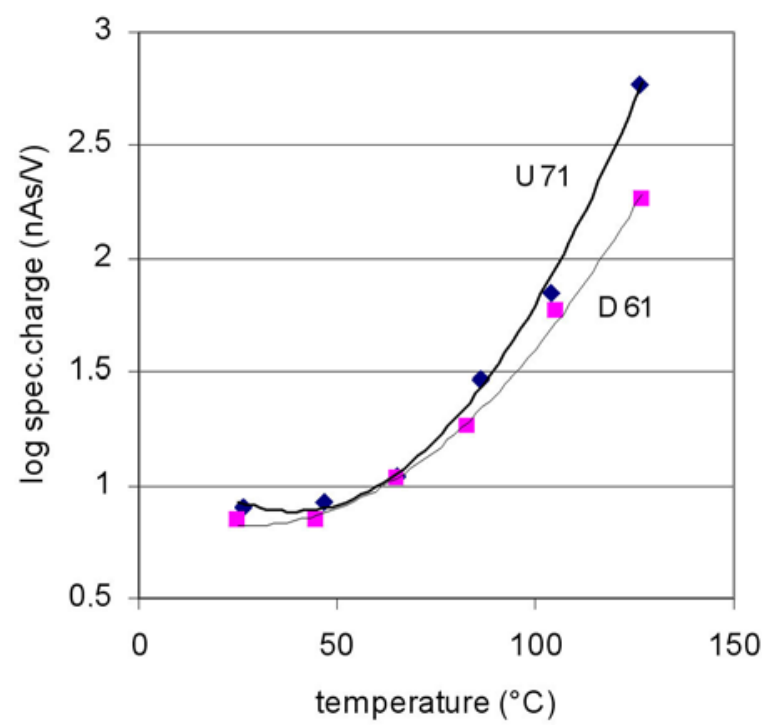

Fig. 12: Comparison of extracted charge (integrated extraction currents)

increase of the extracted charge per unit voltage at injection, and are $\mathrm{d} Q / \mathrm{d} U=C$. This means that the oxide layer behaves like a capacitor which can be charged and discharged.

The temperature dependence of exponent $n$ in eq. 2 is shown in Fig. 11, being equal for both samples inside the measuring errors. The extracted specific charges $C$ for both samples are also equal, as can be seen in Fig. 12. 
Table 3: Comparison of parameters $\left(22^{\circ} \mathrm{C}\right)$

\begin{tabular}{|c|c|c|c|r|}
\hline Sample number & D 61 & D 61 & U 71 & U 71 \\
\hline$\rho(\Omega \mathrm{cm})$ act. energy $(\mathrm{eV})$ & $1.73 \cdot 10^{14}$ & 1.21 & $8.94 \cdot 10^{13}$ & 1.20 \\
\hline$\mu\left(\mathrm{cm}^{2} / \mathrm{Vs}\right)$ act.energy $(\mathrm{eV})$ & $1.44 \cdot 10^{-10}$ & -0.68 & $1.98 \cdot 10^{-10}$ & -0.67 \\
\hline$n\left(\mathrm{~cm}^{-3}\right)$ act.energy $(\mathrm{eV})$ & $2.7 \cdot 10^{14}$ & -0.48 & $2.3 \cdot 10^{14}$ & -0.49 \\
\hline
\end{tabular}

\section{Conclusions}

Comparing the two samples shows that there is a striking similarity of all parameters. Values taken at room temperature are given in Tab. 3, while at higher temperatures they change exponentially, and the exponents are equal.

Taking into account the measurement errors, the sums of the exponents of mobility and carrier concentration are equal to the activation energy of $1.2 \mathrm{eV}$ for both samples, as follows from resistivity $\rho=1 /(e n \mu)$. The resistivity of the doped sample, contrary to expectation, is about twice higher than that of the undoped sample, maybe due to doping atoms filling up the vacancies. The differences of the parameters expressed by their ratio $D / U$ are of the order of $10 \%$. The sum of the ratios for $\mu$ and $n$ is of the same (absolute) value as for $\rho$. Even the injection and extraction currents in the whole temperature range are of the same form and value. At temperatures over $100^{\circ} \mathrm{C}$, a positive zero current (pico-amperemeter without an external voltage source directly connected to the sample electrodes) of similar magnitude, indicating continuing oxidation, is observed in both samples (the extraction current would be negative). Only the relative permittivity is different, being 8.3 and 12.4 for D 61 and $\mathrm{U} 71$, respectively.

From all these found equalities, it follows that Zirconium oxide fits into the group of oxidic semiconductors, where the (low) conductivity is provoked by stoichiometric deviations and not by doping. $\mathrm{ZrO}_{2}$ is an n-type reduction semiconductor, conduction depending on missing oxygen. At higher temperatures, an additional part of ionic conduction by oxygen ions can be observed.

\section{Acknowledgement}

Support for this work from UJP, Praha, a.s., and from MSM grant 680770015 is greatly appreciated. Special thanks are due to Mrs. V. Vrtilková for providing the specimens of measured thickness.

\section{References}

[1] Hintenberger, H.: Z. Phys. 119, 1, (1942).

[2] Frank, H.: J. Nucl. Mater. 340, 119, (2005).

[3] Mott, N. F., Guerney, R. W.: Electronic Processes in Ionic Crystals, Clarendon, Oxford, (1940).

[4] Gould, R. D.: J. Appl Phys., 53, 3 353, (1982).

[5] Frank, H.: Acta Physica Slovaca, 55, 341, (2005).

Prof. RNDr. Helmar Frank

Phone: +420 224358559

E-mail: HelmarFrank@fjfi.cvut.cz

Department of Solid State Engineering

Faculty of Nuclear Sciences and Physical Engineering Czech Technical University

Trojanova 13, Prague 2, Czech Republic 